\title{
Incorporación de Vicia sativa $L$. y fertilización fosfatada en un pastizal del nordeste argentino
}

\author{
Porta, M. ; Hack, C.M. ${ }^{1}$; Castelán, M.E. ${ }^{1}$; Golluscio, R.A. ${ }^{2}$ \\ ${ }^{1}$ Instituto Agrotécnico P.M. Fuentes Godo, Universidad Nacional del Nordeste, Las Heras 727, Resistencia, \\ Chaco, Argentina, Tel: $+54362-4422074$. ${ }^{2}$ Dep. Prod. Anim., Escuela de Agricultura, Universidad de \\ Buenos Aires (UBA) e IFEVA (UBA/CONICET). E-mail: miritaporta@yahoo.com.ar
}

\begin{abstract}
Resumen
Porta, M.; Hack, C.M.; Castelán, M.E.; Golluscio, R.A.: Incorporación de Vicia sativa L. y fertilización fosfatada en un pastizal del nordeste argentino. Rev. vet. 28: 1, 56-61, 2017. En el nordeste argentino, los pastizales megatérmicos constituyen un importante recurso forrajero para la ganadería. Sus principales limitantes son el déficit forrajero invernal y la baja calidad nutritiva. En amplias regiones se agrega, además, el bajo contenido de $\mathrm{P}$ en el suelo y, consecuentemente, en el forraje. Los objetivos de este trabajo fueron evaluar el potencial de la incorporación de una leguminosa y la fertilización fosfatada para aumentar la biomasa de leguminosas y el contenido de fósforo foliar de un pastizal pobre en $\mathrm{P}$ del nordeste argentino (Margarita Belén, Chaco). Se incorporó Vicia sativa y $150 \mathrm{~kg} /$ ha de superfosfato triple en un diseño en bloques en parcelas divididas completamente aleatorizadas $(n=3)$. La aleatorización se realizó asignando a las parcelas principales dos niveles de fertilización fosfatada (sin y con agregado de $\mathrm{P}$ ). A las parcelas secundarias se asignaron dos niveles de agregado de $V$. sativa ( $\sin$ y con agregado de $V$. sativa). La fertilización provocó aumentos en la cobertura y la frecuencia de leguminosas, tanto de las nativas como de la implantada. La incorporación de $V$. sativa y el agregado de $\mathrm{P}$ redundaron en aumentos significativos en la biomasa de leguminosas, pero no en la de gramíneas. Por su parte, el agregado de $\mathrm{P}$ tuvo una influencia favorable en los contenidos de $\mathrm{P}$ foliar de leguminosas y gramíneas, potenciado en ambos casos por el agregado de $V$. sativa. Estos resultados sugieren que la práctica analizada permitiría reducir las principales limitantes para la actividad ganadera en la región. En efecto, el sistema permitió aumentar la cantidad de forraje disponible a fines de invierno-principios de primavera y también aumentar la calidad de la dieta, gracias al aumento del contenido de $\mathrm{P}$ y proteína en la misma.
\end{abstract}

Palabras clave: ganadería, forraje, leguminosas, fósforo, producción de biomasa.

\begin{abstract}
:
Porta, M.; Hack, C.M.; Castelán, M.E.; Golluscio, R.A.: Incorporation of Vicia sativa L. and phosphate fertilization in a grassland of northeast Argentina. Rev. vet. 28: 1, 5661, 2017. Megathermic grasslands constitute the main forage resource for livestock in the great chaco region. In the northeast Argentina, the main constraints for animal feeding are the low quantity and quality of forage, mainly in late winter-early spring. In addition, there are broad regions with low phosphorus content in the soil and in the forage. The aim of this study was to assess the potential of incorporating a leguminous plant and adding phosphate as fertilizer to increase legume biomass and leaf phosphorus content in a grassland poor in phosphorus from northeast Argentina (Margarita Belén, Chaco province). Vicia sativa (20 $\mathrm{kg} / \mathrm{ha})$ and calcium triple superphosphate $(150 \mathrm{~kg} / \mathrm{ha})$ were incorporated in a completely randomized split-plot block design $(\mathrm{n}=3)$. Randomization was carried out by assigning two levels of phosphate fertilization to the main plots (with and without addition of P). Two levels of addition of $V$. sativa (with and without addition of $V$. sativa) were assigned to the secondary plots. Fertilization increased the cover and frequency of both the native and the planted legumes. The incorporation of $V$. sativa and the addition of $\mathrm{P}$ resulted in significant increases in biomass of the legumes but not in grasses. The addition of $\mathrm{P}$ and the addition of $V$. sativa had a favorable influence on the leaf phosphorus contents of legumes and grasses. These results suggest that the incorporation of $V$. sativa and the addition of phosphate as fertilizer
\end{abstract}


would allow to deal with the main factors limiting the livestock activity in the region. Indeed, this practice allowed increasing the amount of available forage in late winter-early spring as well as increasing the quality of the diet, due to the increase in P, and protein content.

Key words: livestock, forage, leguminous, phosphorus, biomass production.

\section{INTRODUCCIÓN}

El gran chaco americano es una vasta planicie que ocupa aproximadamente $1.141 .000 \mathrm{~km}^{2}$, siendo la segunda región boscosa más extensa de América del Sur después de la selva amazónica. El 59\% está en Argentina, el 23\% en Paraguay, el 13\% en Bolivia y el $5 \%$ en Brasil. Se caracteriza por la dominancia de bosques espinosos subtropicales y sabanas húmedas y semiáridas, siendo muy rica en vida silvestre. Se extiende desde latitudes tropicales $\left(18^{\circ} \mathrm{S}\right)$ hasta ambientes subtropicales $\left(31^{\circ} \mathrm{S}\right)$. Presenta una gran variedad de climas y relieves que dan origen a una pluralidad de ambientes que se traduce en una alta biodiversidad ${ }^{13}$.

En el nordeste argentino los pastizales constituyen el principal recurso forrajero para la ganadería en cuanto a superficie. Ocupan aproximadamente el 95\% del área dedicada a esa actividad ${ }^{20}$. Las especies que componen estos pastizales son casi todas gramíneas $\mathrm{C}_{4}$ de crecimiento estival, lo cual determina las dos principales limitantes para la ganadería en la región: la deficiencia de forraje en la época invernal y la baja calidad de la dieta fuera de la estación de crecimiento, particularmente en invierno y primavera temprana ${ }^{18}$.

Otra de las limitantes importantes para el desarrollo de la ganadería en gran parte de esta zona es la deficiencia de fósforo $(\mathrm{P})$ en suelo, que a su vez se manifiesta en bajas concentraciones de fósforo foliar. Esto determina que los animales durante todo el año tengan una dieta pobre en este elemento, lo cual limita su desarrollo ${ }^{16}$. A su vez, tal deficiencia impide la adecuada implantación de leguminosas templadas, que en los trópicos representan el recurso forrajero con mayor potencial para aumentar la producción animal, ya que mejoran los contenidos proteicos de la dieta ${ }^{19}$. Su inclusión es una alternativa económicamente conveniente para reemplazar el uso de concentrados comerciales ${ }^{6,9,17}$.

El objetivo de este trabajo fue evaluar la incorporación de Vicia sativa y la fertilización fosfatada del tapiz vegetal como herramientas para aumentar la biomasa de leguminosas y el contenido de $\mathrm{P}$ foliar de un pastizal natural del noreste argentino.

\section{MATERIAL Y MÉTODOS}

\section{Sitio de estudio}

El trabajo se realizó entre 2007 y 2008 en la localidad de Margarita Belén, Provincia del Chaco, Argentina $\left(27^{\circ} 16^{\prime} \mathrm{S}, 58^{\circ} 57^{\prime} \mathrm{O}\right)$, en un establecimiento de 98 ha, 64 de las cuales estaban divididas en parcelas de 2 ha dedicadas a la cría vacuna. El clima de la zona es subtropical húmedo, con una precipitación media anual de $1200 \mathrm{~mm}$ y una temperatura media mensual entre $14^{\circ} \mathrm{C}$ (julio) y $27^{\circ} \mathrm{C}$ (enero).

Durante el invierno de 2007, tanto la temperatura media como las precipitaciones estuvieron por debajo del promedio histórico. La sequía del invierno de 2007 se repitió durante el verano siguiente.

El suelo predominante es un argiudol típico, con un horizonte superficial franco limoso y un horizonte subsuperficial argílico, moderadamente textural, muy pobre en $\mathrm{P}$ extractable $(3,16 \mathrm{mg} / \mathrm{kg}), \mathrm{N}$ total $(1 \mathrm{~g} / \mathrm{kg}) \mathrm{y}$ materia orgánica $(24 \mathrm{~g} / \mathrm{kg}){ }^{12}$.

La vegetación es herbácea, con un marcado predominio de gramíneas megatérmicas, de ciclo primaveroestival. Presenta un estrato alto dominado por Sorghastrum setosum y uno bajo dominado por Paspalum notatum, acompañado de Paspalum almum, Paspalum plicatulum, Schizachyrium microstachyum, Bothriochloa laguroides y Panicum sp. Las leguminosas nativas presentes son Desmanthus virgatus, Trifolium polymorphum, Vicia epetiolaris y Desmodium incanun.

\section{Descripción del ensayo}

Se seleccionaron tres parcelas de 2 ha que fueron clausuradas al ingreso de animales hasta finalizar el estudio. Cada parcela constituyó un bloque, en un diseño en bloques completos aleatorizados, en parcelas divididas completamente aleatorizadas. En cada parcela de 2 ha se individualizaron cuatro subparcelas de $30 \times 140$ $\mathrm{m}$. La aleatorización se realizó asignando a las parcelas principales dos niveles de fertilización fosfatada ( $\sin$ y con agregado de $\mathrm{P}: \mathrm{P}_{0} \mathrm{y}_{1}$, respectivamente).

A las parcelas secundarias se asignaron dos niveles de agregado de Vicia sativa (sin y con agregado de $V$. sativa: $\mathrm{V}_{0} \mathrm{y}_{1}$, respectivamente). El $\mathrm{P}$ fue aplicado como superfosfato triple de calcio, a razón de $150 \mathrm{~kg} /$ ha y $V$. sativa, fue sembrada a razón de $20 \mathrm{~kg} / \mathrm{ha}$. La siembra y la fertilización se realizaron en mayo de 2007.

En cada subparcela se midieron, a lo largo de una transecta de $100 \mathrm{~m}$, frecuencia y cobertura de $V$. sativa, en los meses donde se encontraba presente, y de leguminosas nativas al inicio, mediados y pre-finalización del ensayo. La medición de cobertura se realizó mediante el método de intersección lineal ${ }^{7}$. Para frecuencia se registró la presencia de leguminosas en una superficie de $0,25 \mathrm{~m}^{2}$ en cada metro de la transecta.

Para evaluar la variación de la biomasa entre septiembre de 2007 y abril de 2008, se tomaron muestras del material vegetal cada 45 días por medio de cortes con tijera, en una superficie de $0,25 \mathrm{~m}^{2}$. Se tomaron 4 muestras al azar dentro de cada subparcela. Se cortó a una altura de $2,5 \mathrm{~cm}$ del suelo en el estrato bajo y $15 \mathrm{~cm}$ en el alto ${ }^{21}$. El material vegetal se llevó a estufa has- 
ta peso constante y se determinó la materia seca total. Luego se separaron manualmente las gramíneas de las leguminosas.

$V$. sativa se encontró solamente en las mediciones de septiembre y octubre, mientras que las leguminosas nativas estuvieron presentes en todas las mediciones. Una vez pesado, el material vegetal fue molido y analizado por medio de una digestión nítrica-perclórica, para determinar el contenido de fósforo foliar en gramíneas y leguminosas, utilizando el método colorimétrico de Murphy-Riley ${ }^{10}$.

\section{Estadísticas}

Los resultados fueron analizados utilizando un ANOVA con un diseño en bloques completos al azar, con un arreglo en parcelas divididas y mediciones repetidas en el tiempo. Las diferencias entre medias fueron analizadas mediante la prueba de Tukey. Las pruebas se realizaron con el programa estadístico INFOSTAT $2006^{5}$.

\section{RESULTADOS}

\section{Frecuencia y cobertura de leguminosas}

Durante septiembre y octubre se registró una alta frecuencia de $V$. sativa (mayor al 50\%) en las parcelas donde esta especie fuera sembrada, independientemen- te de la fertilización fosfatada. En septiembre alcanzó alrededor del $80 \%$ en ambos tratamientos, con diferencias significativas $(p \leq 0,001)$ a favor del tratamiento fertilizado.

En octubre aumentó al 90\% en las parcelas fertilizadas y disminuyó significativamente en las no fertilizadas ( $p \leq 0,001)$ (Figura 1 A). La respuesta a la fertilización fue más marcada en el caso de la cobertura. La misma fue despreciable en ausencia de fertilización y aumentó del 2 al 17\% entre septiembre y octubre solamente en las parcelas fertilizadas $(p \leq 0,001)$ (Figura $1 \mathrm{~B}$ ).

La frecuencia de leguminosas nativas también superó el 50\% hacia el final del experimento y respondió al agregado de P. La presencia de $V$. sativa sólo redujo la frecuencia de leguminosas nativas en la medición inicial en ausencia de fertilización (Figura 2 A). Como en el caso de $V$. sativa, la respuesta a la fertilización fue más marcada aún en términos de cobertura. Alcanzó el 12\% en diciembre en las parcelas fertilizadas sin agregado de $V$. sativa $(p \leq 0.0001)$ (Figura 2 B). Ambas variables alcanzaron su valor máximo en el mes de diciembre.

\section{Biomasa de leguminosas y gramíneas}

Tanto la incorporación de $V$. sativa como el agregado de $\mathrm{P}$ redundaron en aumentos significativos de la biomasa de leguminosas, que incluyó tanto la biomasa
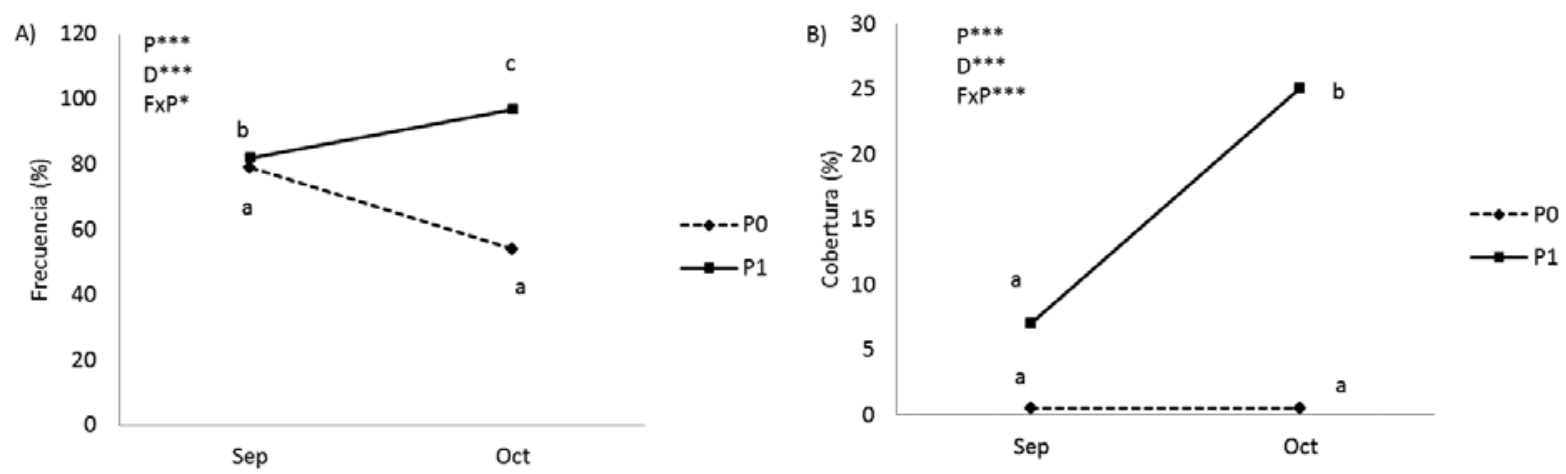

Figura 1. Frecuencia (A) y cobertura (B) de Vicia sativa con y sin agregado de P (P1 y P0 respectivamente). Factores analizados: fecha (D), fertilización fosfatada (P), e interacción DxP. Diferencias entre letras indica diferencias significativas entre las cuatro medidas. ${ }^{*} p<0,05 ; * * p<0,01 ; * * *<0,001$.
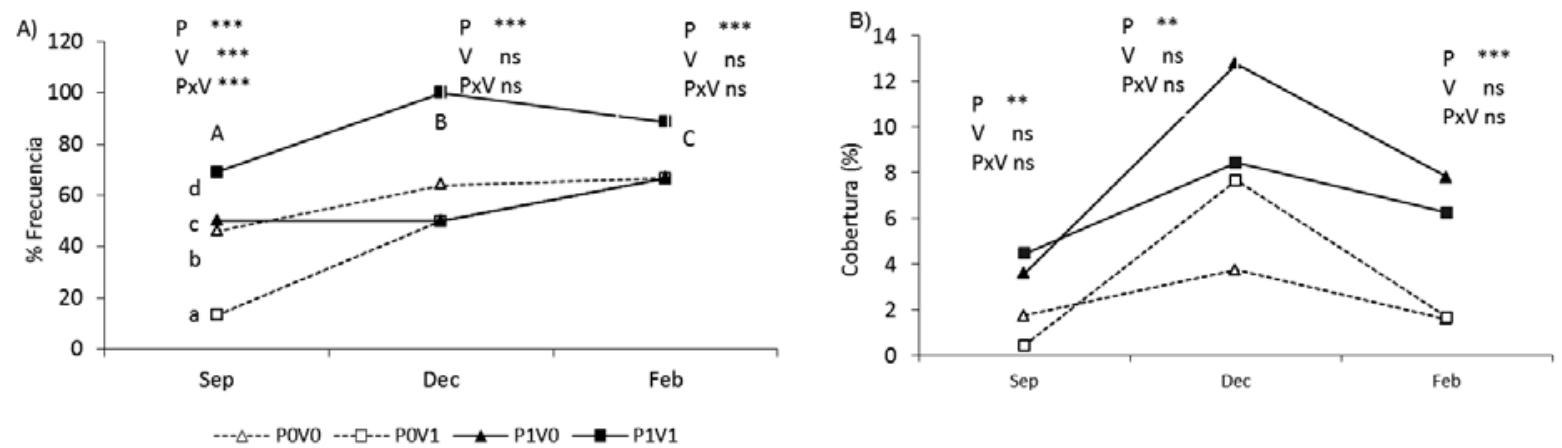

Figura 2. Frecuencia (A) y cobertura (B) de leguminosas nativas. $\mathrm{P}_{0} \mathrm{~V}_{0}$ : sin agregado de $\mathrm{P}$ ni de Vicia sativa; $\mathrm{P}_{0} \mathrm{~V}_{1}$ : sin agregado de $\mathrm{P}$ y con Vicia $; \mathrm{P}_{1} \mathrm{~V}_{0}$ : con agregado de $\mathrm{P}$ y $\sin$ Vicia $; \mathrm{P}_{1} \mathrm{~V}_{1}$ : con agregado de $\mathrm{P}$ y con $V i$ cia. Letras minúsculas indican diferencias dentro de las fechas cuando se encontraron interacciones entre PxV. ${ }^{*} p<0,05 ; * * p<0,01 ; * * * p<0,001$. 

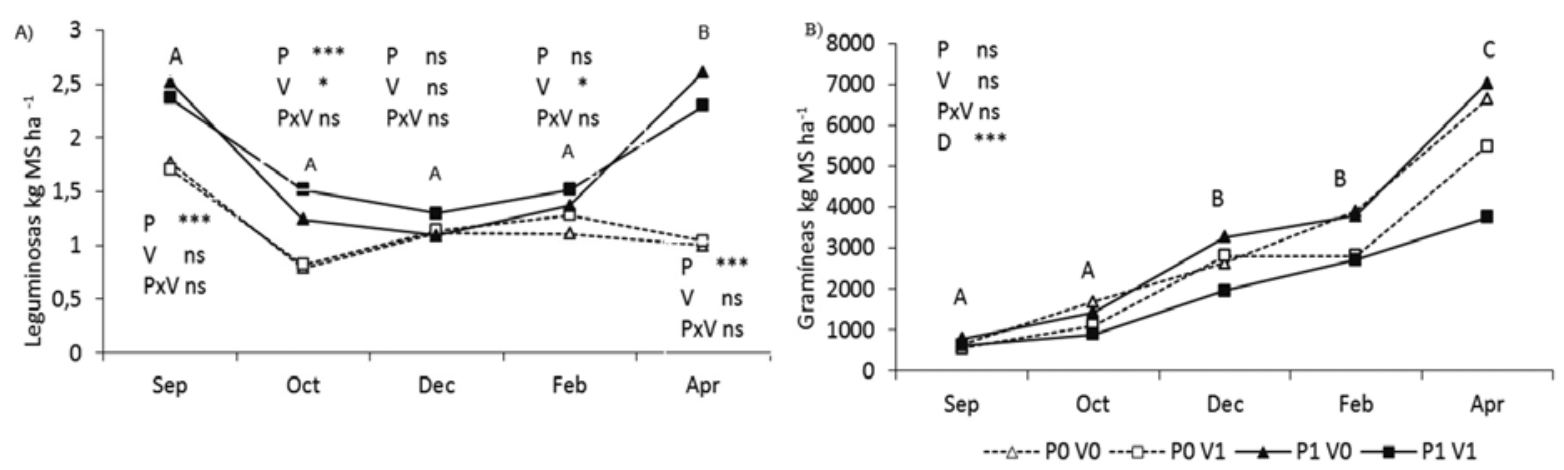

Figura 3. Producción mensual de leguminosas (A) y gramíneas (B) en $\mathrm{kg} \mathrm{MS} / \mathrm{ha} \cdot \mathrm{P}_{0} \mathrm{~V}_{0}$ : $\sin$ agregado de $\mathrm{P}$ y $\sin$ Vicia sativa; $\mathrm{P}_{0} \mathrm{~V}_{1}$ : sin agregado de $\mathrm{P}$ y con Vicia $; \mathrm{P}_{1} \mathrm{~V}_{0}$ : con agregado de $\mathrm{P}$ y $\sin$ Vicia $; \mathrm{P}_{1} \mathrm{~V}_{1}$ : con agregado de $\mathrm{P}$ y con Vicia. Letras mayúsculas indican diferencias entre fechas. ${ }^{*} p<0,05 ; * * p<0,01 ; * * *<<0,001$.
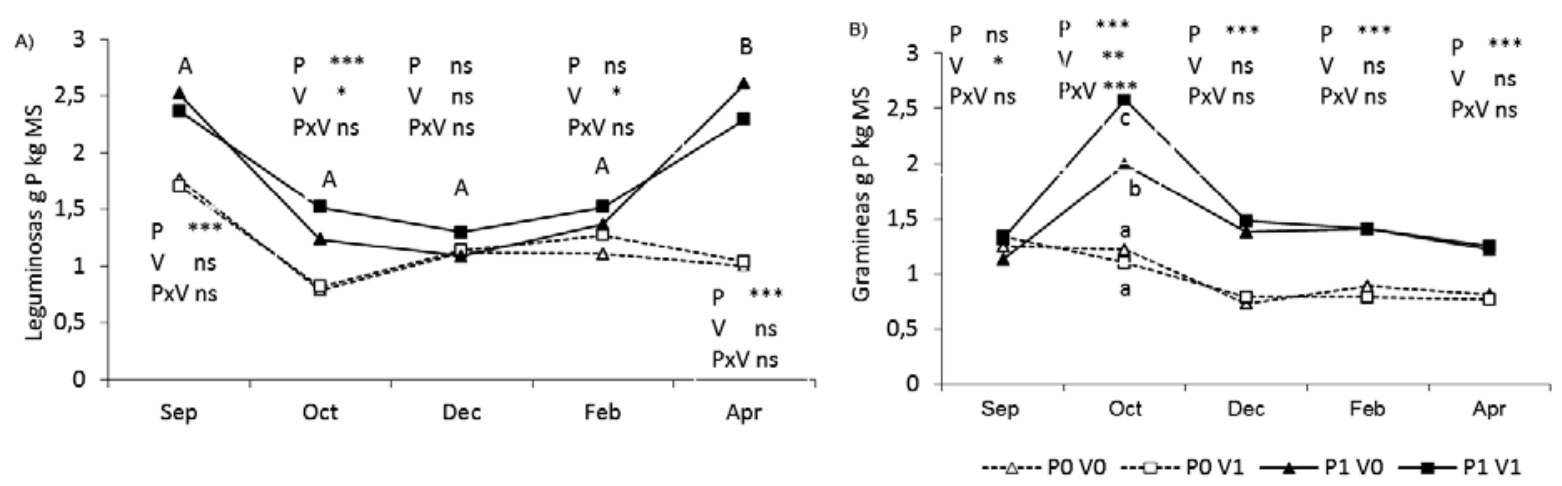

Figura 4. Contenidos de fósforo foliar en leguminosas (A) y gramíneas (B), en g P.kg MS. $\mathrm{P}_{0} \mathrm{~V}_{0}$ : $\sin$ agregado de $\mathrm{P}$ y $\sin$ Vicia sativa; $\mathrm{P}_{0} \mathrm{~V}_{1}$ : sin agregado de $\mathrm{P}$ con Vicia; $\mathrm{P}_{1} \mathrm{~V}_{0}$ : con agregado de $\mathrm{P} \sin$ Vicia; $\mathrm{P}_{1} \mathrm{~V}_{1}$ : con agregado de $\mathrm{P}$ con Vicia. Letras mayúsculas indican diferencias entre fechas, minúsculas indican diferencias dentro de las fechas cuando se encontraron interacciones entre PxV. ${ }^{*} p<0,05 ;{ }^{* *} p<0,01 ; *^{* *} p<0,001$.

de las leguminosas nativas como la de $V$. sativa (Figura 3 A). Sin embargo, el efecto promotor del agregado de $V$. sativa fue más importante en las dos primeras mediciones, mientras la especie se encontraba fenológicamente activa, que al final del experimento.

Un hallazgo novedoso fue que el agregado de $V$. sativa incrementó la biomasa de leguminosas en general, incluso en diciembre, cuando dicha especie ya no se encontraba activa. Por su parte, el efecto promotor de la fertilización fosfatada se hizo más evidente hacia el final del experimento, cuando sólo se encontraban las leguminosas nativas (Figura $3 \mathrm{~A}$ ).

Por su parte, la biomasa de las gramíneas aumentó sostenidamente a lo largo de las cinco fechas de corte. Se trató de especies $\mathrm{C}_{4}$, de crecimiento primavero-estival, con valores promedio que fueron desde $652 \mathrm{~kg} \mathrm{MS} /$ ha en septiembre hasta $5727 \mathrm{~kg} \mathrm{MS} / \mathrm{ha}$ en abril (Figura 3 B). No se encontraron efectos de la fertilización fosfatada, del agregado de $V$. sativa, ni de ninguna de las interacciones analizadas.

Contenido de fósforo foliar de leguminosas y gramíneas

$\mathrm{El}$ agregado de $\mathrm{P}$ al suelo tuvo un efecto promotor sobre el contenido de $\mathrm{P}$ foliar en las leguminosas y lo mismo ocurrió con el efecto temprano (octubre) de la incorporación de V. sativa. Sin embargo, el efecto promotor del agregado de $V$. sativa sobre el contenido de $\mathrm{P}$ foliar de las leguminosas nativas se reiteró también en febrero, cuando $V$. sativa ya había culminado su ciclo (Figura 4 A).

En las parcelas no fertilizadas el contenido de $\mathrm{P}$ foliar de las gramíneas disminuyó sostenidamente a lo largo del experimento. En cambio, en las fertilizadas, el contenido de $\mathrm{P}$ foliar de las gramíneas mostró un marcado incremento en octubre, potenciado por la incorporación de $V$. sativa. A partir de esa fecha, el contenido de $\mathrm{P}$ foliar de las gramíneas también se redujo pero siempre se mantuvo por encima del valor de las parcelas no fertilizadas (Figura 4 B).

\section{DISCUSIÓN}

Las leguminosas se vieron favorecidas por el agregado de $\mathrm{P}$, especialmente en términos de cobertura, tanto en el caso de $V$. sativa (más evidente hacia final del ciclo de dicha especie, Figuras 1 A y B), como en las leguminosas nativas (Figuras 2 A y B). Como consecuencia, se incrementó significativamente la biomasa de leguminosas (Figura 3 A). Este efecto promotor del agregado de $\mathrm{P}$ ha sido reiteradamente descripto en la bibliografía $1,2,3,8,14$. 
Tal incremento en la biomasa de leguminosas subtropicales (por ejemplo en Stylosanthes scabra) en respuesta a la fertilización fosfatada, no se debería a un aumento del número de plantas por $\mathrm{m}^{2}$ sino a un aumento del tamaño de las plantas ${ }^{3}$. En cambio, la biomasa de las gramíneas no registró cambios en respuesta a la fertilización fosfatada.

Tal ausencia de respuesta a la fertilización fosfatada en la producción de gramíneas fue comparable con los resultados obtenidos por nosotros en suelos ultisoles del nordeste de la Provincia de Corrientes, Argentina, donde se probaron distintas dosis de $\mathrm{P}$ en pastizales y no se encontraron diferencias significativas en la producción de MS (Porta M, Hack CM, Tomei CE, Ciotti EM, Castelán ME, Agrotecnia 18: 3-5. 2008).

Por otro lado, en este trabajo la adición de fertilizante fosfatado permitió elevar alrededor del $40 \%$ los contenidos de $\mathrm{P}$ foliar, tanto en leguminosas (36\%) como en gramíneas (40\%) (Figura 4). El incremento en el contenido de $\mathrm{P}$ foliar de leguminosas nativas en respuesta al agregado de $\mathrm{P}$, al estar acompañado por un aumento de su biomasa, sugiere que, pese a estar adaptadas a crecer en suelos deficientes en $\mathrm{P}$, se encontraban limitadas por dicho elemento ${ }^{11}$.

En cambio, el incremento en el contenido de $\mathrm{P}$ foliar registrado en las gramíneas de las parcelas fertilizadas no se tradujo en un incremento de producción de MS, lo cual sugiere que hubo "consumo de lujo". Ese fenómeno se produce cuando el crecimiento de las plantas es inferior al incremento en la absorción de nutrientes, los que son reservados para sus próximos rebrotes ${ }^{4,15}$.

Los efectos positivos de la presencia de $V$. sativa sobre el contenido de fósforo foliar de las leguminosas nativas (en febrero) y de las gramíneas (en setiembre y octubre) (Figuras 4 A y B, respectivamente) se podrían atribuir a que el sistema radical de $V$. sativa segrega ácidos orgánicos que remueven el $\mathrm{P}$ del suelo ${ }^{22}$.

Se concluye que los incrementos obtenidos en la biomasa de leguminosas y el contenido de $\mathrm{P}$ de gramíneas y leguminosas en las parcelas fertilizadas y enriquecidas con $V$. sativa a comienzos de la primavera, sugieren que la práctica puede ser promisoria como estrategia para remover la limitación impuesta por la baja disponibilidad forrajera y la baja calidad de los pastizales en esa época clave del ciclo reproductivo de los rodeos de cría vacuna de zonas del nordeste argentino deficientes en $\mathrm{P}$ edáfico.

\section{REFERENCIAS}

1. Berardo A, Marino M. 2000. Efecto de la fertilización fosfatada sobre la disponibilidad de fósforo y su relación con la producción de forraje en molisoles del sudoeste bonaerense. II-Alfalfa. Congreso Argentino de Ciencias del Suelo, Mar del Plata, Argentina, Com. III, Trabajo $\mathrm{N}^{\circ} 6$.

2. Bono A, Montoya J, Babinec F, Lescano P, Buschiazo D. 1997. Fertilización combinada en las zonas semiárida y subhúmeda. Rev Agromercado 1: 23-26.
3. Coates DB, Kerridge PC, Miller CP, Winter WH. 1990. Phosphorus and beef production in northern Australia. The effect of phosphorus on the composition, yield and quality of legume-based pasture and their relation to animal production. Trop Grasslands 24: 209-220.

4. Chapin SF. 1980. The mineral nutrition of wild plants. Annual Review Ecol \& System 11: 233-260.

5. Di Rienzo JA, Balzarini M, Casanaves F, Gonzáles L, Tablada M, Robledo CW. 2006. Infostat Profesional. Versión 2006, Facultad de Ciencias Agropecuarias, Universidad Nacional de Córdoba, Argentina.

6. Eusse BJ. 1994. Pastos y Forrajes Tropicales, 3ra. Ed., Banco Ganadero, Santa Fé de Bogotá, Colombia, p. 320420.

7. Greig-Smith P. 1983. Quantitative Plant Ecology, 3rd. Ed., University of California Press, Berkeley, USA, 359 p.

8. Hu FD, Jones R.J. 2001. Competition in pots between two tropical legumes (Stylosanthes hamata and S. scabra) and two tropical grasses (Urochloa mosambicensis and Bothriochloa pertusa) at two phosphorus fertilizer levels. Trop Grasslands 35: 34-42.

9. Humphreys LR. 1991. Tropical pasture utilization, Ed. Cambridge University Press, Cambridge, UK, 172 p.

10. Jackson ML. 1964. Análisis químicos de los suelos, $2^{\circ} \mathrm{Ed}$., Editorial Omega, Barcelona, 666 p.

11. James PM, Taylor J, Interrante SM. 2005. Herbage and seed from texan native perennial herbaceous legumes. Rang Ecol \& Manag 58: 643-651.

12. Ledesma LL, Zurita JJ. 1995. Los suelos de la Provincia del Chaco, Ed. INTA, Argentina, 164 p.

13. Maldonado P, Hohne E. 2006. Atlas del Gran Chaco Americano, Publ. Programa de Acción Subregional para el Desarrollo Sostenible del Gran Chaco Americano; GTZ/ Ministerios de Educación de Argentina, Bolivia y Paraguay, $96 \mathrm{p}$.

14. Morón A. 2000. Alfalfa: fertilidad de suelos y estado nutricional en sistemas agropecuarios de Uruguay. Plant Nutr Inst 8: 1-6.

15. Oyarzabal M, Oesterheld M. 2009. Phosphorus reserves increase grass regrowth alter defoliation. Oecologia 15: 717-724.

16. Pizzio RM, Delfino D, Rivero L, Fernandez JG. 2005. Mejoramiento del campo natural en el Departamento de Curuzú Cuatiá. Boletín Noticias y Comentarios (INTA Ediciones), № 399.

17. Plaza HC, Lascano CE. 2005. Utilidad de Cratylia argentea en las ganaderías de doble propósito del piedemonte de los llanos orientales de Colombia. Past Tropic 27: 65-72.

18. Royo Pallares O, Beretta E, Maraschin G. 2005. The South American campos ecosystem. In: Grasslands of the World. FAO, capítulo 5.

19. Skerman PJ, Cameron DG, Riveros F. 1992. Leguminosas forrajeras tropicales, Prod \& Protec Veget 2: 1-635.

20. Tomei CE. 1995. Manejo racional de los campos naturales para mejorar la producción ganadera en la Región Chaqueña. Anales Jorn Intern de Actualiz Ganad Subtrop, Chaco (Argentina), p. 61-66. 
21. Toledo JM, Schultze-Kraft R. 1982. Metodología para la evaluación agronómica de pastos tropicales, Publ. Red Internacional de Evaluación de Pastos Tropicales, Cali, Colombia, p. 91-109.

22. Vélez J, Arteaga G, Castillo J, Menjivar JC. 2006. Variaciones en el $\mathrm{pH}$ de la rízofera y en el porcentaje de materia seca de Vicia sativa al aplicar dos fuentes fosfatadas de baja solubilidad en un Andisol del departamento de Nariño, Colombia Acta Agronómica 2: 23-28.

\section{SJR sclmase Journal \& Country Rank}

\section{Revista Veterinaria aumentó su índice de impacto}

Noticias de Scimago Research Group (Scimago Journals \& Country Ranks, Scopus-Elsevier) comunican que la publicación de nuestra casa, Revista Veterinaria, aumentó su índice de impacto. El índice SJR mide la influencia científica (impacto) del artículo de una revista, expresando cuán importante es el "artículo promedio" de la publicación en la discusión científica global (sistema Thomson Reuters).

Para nuestra revista, tal indicador había sido de 0,03 entre 2008 y 2011, aumentando a 0,05 en 2012, a 0,11 en 2013 y a 0,108 en 2015, último período evaluado. El aumento fue del $370 \%$. Asimismo, surge para nuestra publicación un sostenido descenso del indicador que relaciona "citas versus autocitas", demostrando que los autores de los artículos están abandonando la práctica de citar sus propias publicaciones anteriores.

Por último, se advierte que según este portal, nuestra "Revista Veterinaria" continúa siendo la única publicación de esta rama de la ciencia que posee índice de impacto en Argentina. Para el resto del cono sur tal distinción recae en Brasil, Chile, Colombia y Venezuela. No registran índice de impacto las revistas de veterinaria de Bolivia, Paraguay, Uruguay, Perú y Ecuador. En revistas de veterinaria, a nivel mundial el mayor índice de impacto (1,22 puntos) recae en Veterinary Microbiology (Amsterdam, Holanda). 